\title{
Inversion of microscopic lock-in thermograms in the presence of emissivity contrast
}

\author{
by O. Breitenstein ${ }^{1}$ and F. Altmann ${ }^{2}$ \\ ${ }^{1}$ Max Planck Institute of Microstructure Physics, Weinberg 2, D-06120 Halle, Germany; ${ }^{2}$ Fraunhofer Institute for \\ Mechanics of Materials, Heideallee 19, D-06120 Halle, Germany
}

\begin{abstract}
None of the conventionally displayed images of lock-in thermography (in-phase or $0^{\circ}$ image, out of phase or $-90^{\circ}$ image, amplitude image, phase image) is able to become inverted in the presence of emissivity contrast. The phase signal is inherently emissivity-corrected, but here contributions of different heat sources superimpose very nonlinearly, which prevents any meaningful deconvolution. It is shown that the " $0 \%-90^{\circ}$ image, as an alternative kind of representation of lock-in thermography images, being also inherently emissivity-corrected, is able to be inverted for obtaining e.g. the lateral power distribution in an electronic device. Blackening the surface by colloidal bismuth also may remove the emissivity contrast.
\end{abstract}

Keywords: lock-in thermography, emissivity, image inversion, deconvolution, electronic devices

\section{Introduction}

Lock-in thermography (LIT, often also called thermal wave imaging), which is a very successful technique in non-destructive evaluation (NDE) since many years [1], was in the last years increasingly used for failure analysis in electronic devices [2,3]. The goal of electronic device failure analysis is not to detect subsurface structures, as for NDE, but rather to image electrically activated local heat sources in these devices. Until now LIT has been used successfully e.g. for investigating shunts in solar cells and for failure analysis (FA) of integrated circuits (ICs). Many types of failures in ICs, like gate oxide brakdowns, latchups, or line shorts, are leading to local heat sources, if the supply voltage is applied to the device. Even the normal operation of these devices is leading to internal electrically stimulated local heat sources. Therefore, microthermal inspection has been a standard FA tool for IC inspection since many years. Until recently only techniques like liquid crystal microscopy [4] or fluorescent microthermal imaging (FMI) [5] have been used in this field, rather than infrared (IR) imaging. There are two main reasons for that: 1 . The spatial resolution of thermal IR imaging is limited by the used wavelength of typically 3-5 $\mu \mathrm{m}$ to the order of $5 \mu \mathrm{m}$. 2. The IR images are heavily corrupted by the so-called 'emissivity contrast'. Note that the top layers of ICs are partly covered with metal lines, which show a much smaller IR emissivity than the surrounding silicon material. Thus, these lines appear dark in an IR image, compared to the surrounding silicon material of the same temperature, and this 'emissivity contrast' often dominates over the actually interesting thermal contrast.

The introduction of lock-in thermography (LIT) shows a dramatic improvement of the detection sensitivity and opens new possibilities to correct for the emissivity contrast, as compared to steadystate thermography. For example, the phase image, which is actually a measure of the time delay of the periodic temperature modulation compared to the applied voltage pulses, is inherently emissivitycorrected. Thus, in the presence of emissivity contrast, the phase image is much more meaningful for imaging internal local heat sources than the amplitude image. LIT is about two orders of magnitude more sensitive than previous microthermal FA techniques [4,5], therefore LIT has gained new interest in the FA community, in spite of its limited spatial resolution. 
Another general problem of thermal analysis is the thermal blurring effect, which is due to the inevitable lateral heat conductivity in the investigated material. Though, due to the dynamic nature of LIT, lateral heat diffusion is already suppressed in LIT to a certain degree, especially for microscopic investigations the thermal blurring often still disturbs. Note that the characteristic decay length of thermal waves is the thermal diffusion length $\Lambda$, which is about $1 \mathrm{~mm}$ in silicon for a typical lock-in frequency of $30 \mathrm{~Hz}$ and is reducing with $1 / \sqrt{ } \mathrm{f}_{\text {lock-in }}$ [6]. Especially for microsocopic investigations, having a pixel resolution below $10 \mu \mathrm{m}$, the detected thermograms may considerably deviate from the acually interesting lateral power density distribution. This blurring effect may be reduced by working at higher lock-in frequencies, which, however, is always technically limited.

Mathematically, the blurring effect can be described by a convolution process. The (complex) temperature response $T(x, y)$ around a certain (real) lateral power distribution $P(x, y)$ is given by a mathematical convolution between this power distribution and the 2-dimensional (complex) point spread function $\operatorname{PSF}(x, y)[2,3]$. If this PSF is known, the power distribution may be calculated from the measured lock-in thermography image $T(x, y)$ by applying an appropriate image deconvolution procedure. This procedure is also called 'inversion', since it inverts the thermal blurring effect. In this contribution, first we present some simulations, showing that the inversion of conventional lock-in thermograms is not meaningful in the presence of emissivity contrast. Even the phase image, which is inherently emissivity-corrected, is not able to become inverted, since it depends very non-linearly on the power of different heat sources. Then we show that the $10 \%-90^{\circ}$ image, as a special kind of representing LIT images, is able to become inverted for microscopic applications, even in the presence of emissivity contrast. Finally, we are presenting a novel technique of blackening the surface of ICs for removing the emissivity contrast.

\section{Experimental}

All LIT results shown in this contribution were obtained by using the TDL $384 \mathrm{M}$ 'Lock-in' thermography system by Thermosensorik GmbH (Erlangen). This LIT system is based on a Stirlingcooled $384 \times 288$ pixel mercury-cadmium-telluride focal plane array, which is working in the mid IR range $(3-5 \mu \mathrm{m})$ and shows a room temperature noise level (NETD) of $20 \mathrm{mK}$ at a full frame rate of $120 \mathrm{~Hz}$. For performing microscopic investigations, it may be equipped with a $\times 2.5$ or a $\times 5$ microscope objective, leading to a pixel resolution of $10 \mu \mathrm{m}$ and $5 \mu \mathrm{m}$, respectively. This system is working in synchronous operation mode, hence the lock-in trigger is derived from the frame trigger of the free-running IR camera. The modulation of the internal heat sources in the investigated devices is performed by periodically pulsing the supply voltage of the devices (on and off) with a duty cycle of 1:1. The lock-in correlation is performed as a two-phase sine / cosine correlation on-line with the resulting image being updated and displayed every 10 seconds. The system allows to display one of the primary signals (inphase $/ 0^{\circ}$ signal or out-of-phase $/-90^{\circ}$ signal) or the amplitude or the phase signal at choice. A constant systematic phase error may be corrected.

The simulations and deconvolutions presented here were performed by using the DECONV software, which was developed by the authors [3]. This software allows to simulate any type of lock-in thermogram from a given lateral power distribution, which may be at the surface or at a constant depth of the sample. The sample may be assumed to be either infinitely thin, infinitely thick, or to have a welldefined constant thickness, but it is assumed to be laterally and depth-homogeneous. For a finite sample thickness, the calculation includes mirror heat sources to account for the two surfaces. The main task of the DECONV software is to deconvolute measured or simulated lock-in thermograms by using two alternative approaches, which are the Fast Fourier Transform (FFT) based deconvolution and the Constraint Iterative technique, for details see [2,3]. In both cases, a compromise between the acheaved spatial resolution and the inevitable degradation of the signal-to-noise ratio may be chosen. A regularisation (noise reduction) procedure for the input images by using a Wavelet or a JPEG based procedure is possible, but was not used for the examples introduced here. 
Proc. Vth International Workshop, Advances in Signal Processing for Non Destructive Evaluation of Materials Québec City (Canada),2-4 Aug. 2005. @ X. Maldague ed., É. du CAO (2006), ISBN 2-9809199-0-X

\section{Simulations}

An IC containing microscopic heat sources at the surface can be modelled to a good accuracy as a homogeneous sample of infinite thickness. In this case, the LIT point spread function, which is the thermal response to an oscillating point heat source at the surface, is well-known [6]:

$$
\operatorname{PSF}(r, t)=\frac{A}{r} \exp \left(-r \sqrt{\frac{i \omega c_{p} \rho}{\lambda}}\right) \exp (i \omega t)=\frac{A}{r} \exp \left(\frac{-r}{\Lambda}\right) \exp \left(i\left(\omega t-\frac{r}{\Lambda}\right)\right)
$$

$$
\text { with } r=\sqrt{x^{2}+y^{2}} \text { and } \quad \Lambda=\sqrt{\frac{2 \lambda}{\rho c_{p} \omega}} \quad \text { (thermal diffusion length) }
$$

( $r$ = distance from point source, $A=$ amplitude factor; depending on the power, $\omega=2 \pi f_{\text {lock-in }}, i=\sqrt{ }-1$, $t=$ time, $c_{p}=$ specific heat, $\rho=$ density, $\lambda=$ heat conductivity). The local in-phase (or $\left.0^{\circ}\right)$ contribution of the PSF, which is most appropriate for deconvolution [2], is according to (1):

$$
\operatorname{PSF}^{0^{\circ}}(\mathrm{x}, \mathrm{y})=\frac{\mathrm{A}}{\sqrt{\mathrm{x}^{2}+\mathrm{y}^{2}}} \exp \left(\frac{-\sqrt{\mathrm{x}^{2}+\mathrm{y}^{2}}}{\Lambda}\right) \cos \left(\frac{-\sqrt{\mathrm{x}^{2}+\mathrm{y}^{2}}}{\Lambda}\right)
$$

If $\varepsilon(x, y)$ is the lateral distribution of the emissivity (emissivity image), the $0^{\circ}$-image resulting from a LIT measurement of a lateral power distribution $\mathrm{P}(\mathrm{x}, \mathrm{y})$ calculates to:

$$
\mathrm{T}_{\varepsilon} 0^{\circ}(\mathrm{x}, \mathrm{y})=\varepsilon(\mathrm{x}, \mathrm{y}) \int_{\mathrm{x}, \mathrm{y}} \mathrm{P}(\mathrm{x}, \mathrm{y}) \operatorname{PSF}\left(\mathrm{x}-\mathrm{x}^{\prime}, \mathrm{y}-\mathrm{y}^{\prime}\right) \mathrm{dx^{ \prime }} d \mathrm{y}^{\prime}
$$
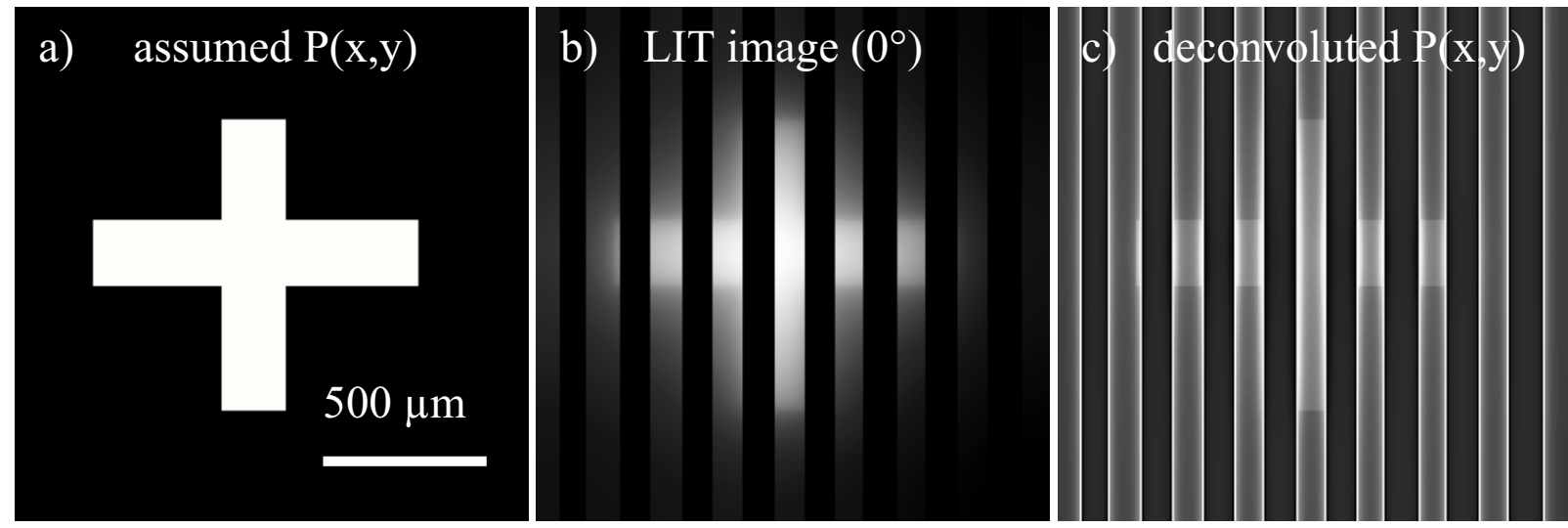

Fig. 1: (a) Assumed power distribution, (b) simulated $0^{\circ}$ lock-in thermogram, (c) result of deconvolution

This simulation has been performed assuming a cross-shaped power distribution, $\mathrm{f}_{\text {lock-in }}=10 \mathrm{~Hz}$, and an emissivity image consisting from vertical stripes with an emissivity contrast of 1:0.1, which mimic vertical metallic lines at the surface. The result in Fig. 1 shows that the power cross appears blurred in the LIT image, and that the assumed metal lines appear dark, as expected. This image was formally deconvoluted for revealing the power distribution, leading to image (c). While a deconvolution of a thermogram of this power distribution with a homogeneous emissivity of $\varepsilon(x, y)=1$ exactly revealed the assumed power distribution (a), the result in (c) is strongly corrupted by the emissivity contrast, hence it is nearly meaningless. This simulation demonstrates that, in the presence of a strong emissivity contrast, which is typical for ICs, conventional deconvolution of LIT images does not work. In the following two sections, two solutions to this problem are introduced. 
Proc. Vth International Workshop, Advances in Signal Processing for Non Destructive Evaluation of Materials Québec City (Canada),2-4 Aug. 2005. ( X. Maldague ed., É. du CAO (2006), ISBN 2-9809199-0-X

\section{Deconvolution using $0 \%-90^{\circ}$ image}

The usual way to suppress an emissivity contrast in IR imaging is to cover the surface with a "black" layer having a high and homogeneous emissivity in the wavelength range of the IR camera. However, for inspecting microscopic devices like ICs, this measure is only hardly applicable. The thickness of usual paint layers is between 20 and $200 \mu \mathrm{m}$, which is already large against the typical pixel resolution of 5 to $10 \mu \mathrm{m}$. Note that only the surface of the black layer emits light, and the heat spreads in all 3 dimensions within the black layer. Therefore the application of this layer would drastically reduce the spatial resolution. The application of an especially thin black layer is described in the following section. The question remains, in which way LIT images of an uncovered IC are able to become deconvoluted. For answering this question, let us first consider the different ways to display LIT images.

The two primary images of a LIT experiment are the $0^{\circ}$ and the $-90^{\circ}$ image, representing the magnitudes of the temperature modulation component being in-phase with the applied bias pulses (the $0^{\circ}$ image) and of the component being out-of-phase to the bias pulses (the $-90^{\circ}$ image). The $-90^{\circ}$ image is usually chosen instead of the $+90^{\circ}$ one (which actually would be the imaginary part), since the latter is essentially negative. From these two images, the phase-independent amplitude image $A$ and the phase image $\Phi$ may easily be calculated:

$$
\mathrm{A}=\sqrt{\left(\mathrm{T}^{0^{\circ}}\right)^{2}+\left(\mathrm{T}^{-90^{\circ}}\right)^{2}} \quad \Phi=\arctan \left(\frac{-\mathrm{T}^{-90^{\circ}}}{\mathrm{T}^{0^{\circ}}}\right)
$$

The conventional way to display LIT results is to use one of these four representations, which show different properties [2]. The $0^{\circ}$ image shows the best spatial resolution, which may be much better than the thermal diffusion length $\Lambda$, whereas the resolution of the $-90^{\circ}$ image is much poorer and in the order of $\Lambda$. If there are different nearby lying heat sources in the device with their thermal signals overlapping, their contributions add up linearly only for these two primary components. The amplitude image $A$ is a vector addition of these components according to (4), hence it also shows a good spatial resolution with a stronger halo around than the $0^{\circ}$ image, but here contributions of different heat sources don't add up exactly linearly anymore. The $0^{\circ}$, the $-90^{\circ}$, and the amplitude images are all modulated by the emissivity contrast. In contrast, the phase image $\Phi$ shows inherent emissivity correction, since both the $0^{\circ}$ and the $-90^{\circ}$ image are modulated by the emissivity (see (4)). On the other hand, the spatial resolution of the phase signal is only in the order of $\Lambda$, and overlapping contributions of the phase signal add up very nonlinearly. Note that the two basic conditions for the application of image deconvolution are the absence of emissivity contrast and that image contributions from different sources have to add up linearly. According to these two conditions, none of the conventionally displayed LIT images is able to become deconvoluted successfully.

In the following we will shows that the $10^{\circ} /-90^{\circ}$ image, as another kind of representation of LIT images, can be deconvoluted, at least for microscopic investigations. The $0 \%-90^{\circ}$ signal is related to the inverse of the phase signal $\Phi$. Like the phase signal, it is inherently emissivity-corrected, but in contrast to this it shows a better spatial resolution, just like the $0^{\circ}$ signal. Note that, for a point heat source at the surface, the $0^{\circ}$ signal and the amplitude signal are diverging in source position, but the $-90^{\circ}$ and the phase signal are not. This is the reason for the poor spatial resolution of $-90^{\circ}$ and phase images. In microscopic regions, hence for lateral distances smaller than $\Lambda$, the thermal $-90^{\circ}$ signal can be taken to be 'nearly constant'. Since this signal is modulated by the emissivity, the $-90^{\circ}$ signal is in good approximation proportional to the emissivity, hence the $0^{\circ} /-90^{\circ}$ signal is an 'emissivity-corrected $0^{\circ}$ signal'. As such, it can be deconvoluted, as shown below. Moreover, this kind of representation has the advantage of having a much better spatial resolution than the phase signal. So, even if no image deconvolution is intended, it is often more useful to image the $0 \%-90^{\circ}$ signal than the phase signal. 
Fig. 2 shows the $0^{\circ}$ image, the $-90^{\circ}$ image, the $0^{\circ} /-90^{\circ}$ image, and the power image deconvoluted from the $0 \%-90^{\circ}$ image, of an operational amplifier $(\mu \mathrm{A} 741)$ in normal operation with the supply voltage pulsed at $f_{\text {lock-in }}=10 \mathrm{~Hz}$. The total dissipated power is about $6 \mathrm{~mW}$, the thermal signals are in the order of some $\mathrm{mK}$. While the $-90^{\circ}$ signal (b) displays essentially the emissivity contrast, in the $0^{\circ}$ image (a) a certain inhomogeneity is already visible. However, also this image is dominated by the emissivity contrast. All local heat sources are easily visible in the $0^{\circ} /-90^{\circ}$ image (c), which is perfectly emissivitycorrected. In the deconvoluted power distribution (d) the spatial resolution is further improved, but the signal-to-noise ratio is degraded. This degradation, which is inherent to any kind of image deconvolution, is especially remakable in the very low emissivity region at the bottom left.
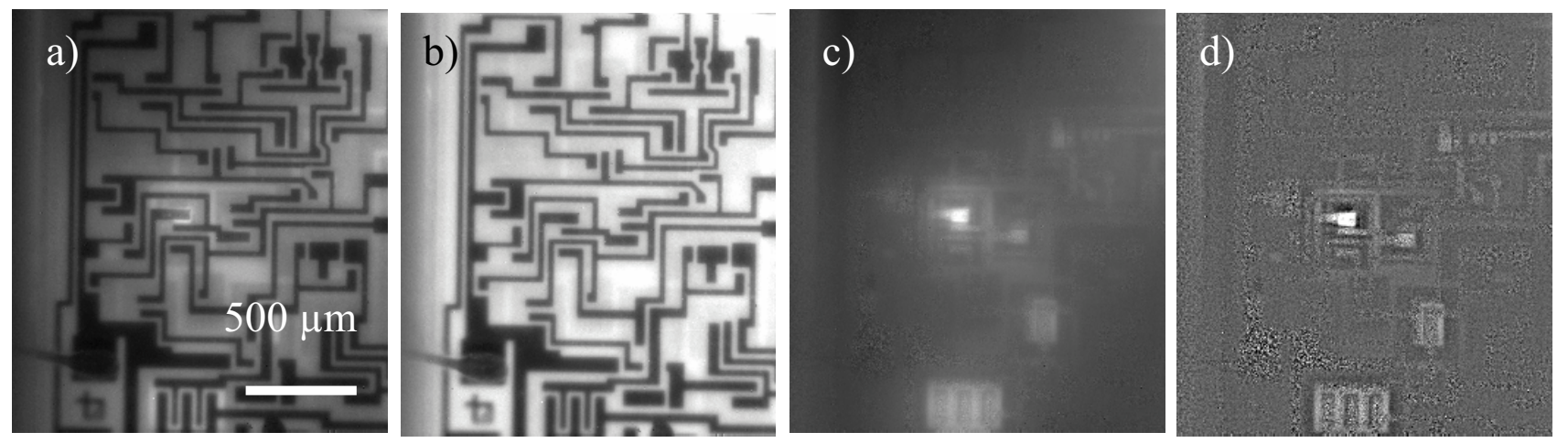

Fig. 2: (a) $0^{\circ}$ image, (b) $-90^{\circ}$ image, (c) $0^{\circ} / 90^{\circ}$ image, and (d) power distribution deconvoluted from (c) of an operational amplifier in normal (pulsed) operation

\section{Blackening the surface with colloidal bismuth}
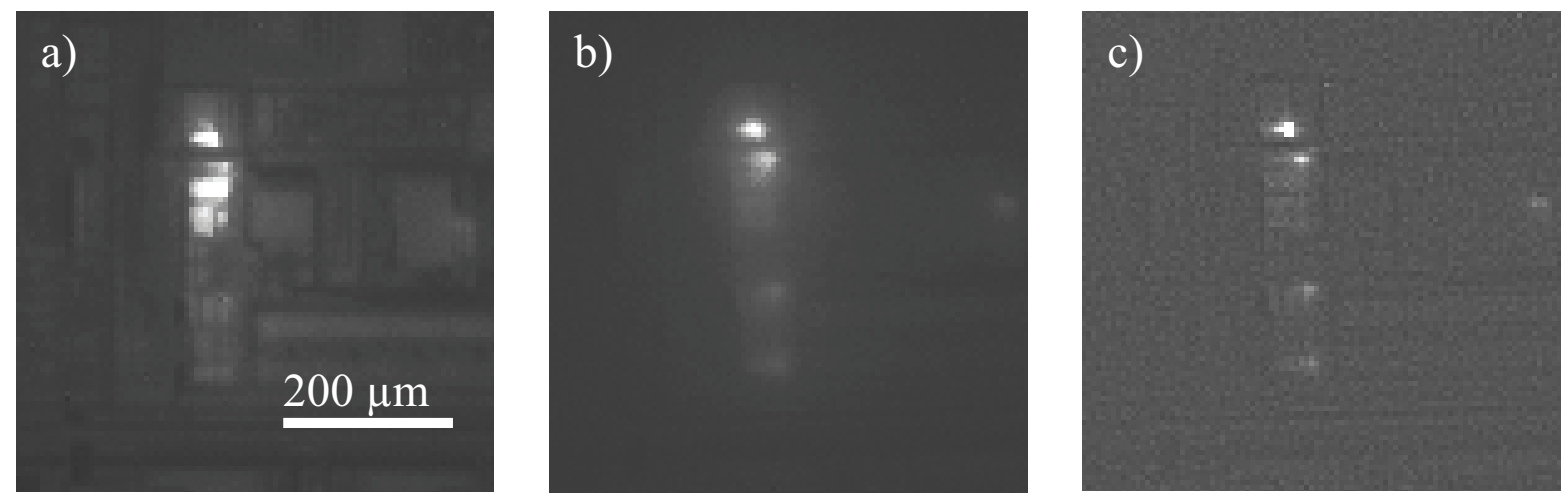

Fig. 3: (a) $0^{\circ}$ image of the uncovered surface, (b) $0^{\circ}$ image of the blackened surface, and (c) power distribution deconvoluted from (b) of a CMOS IC containing failure-induced heat sources

It was mentioned in the previous section that layers of usual paints are too thick to be applied to microscopic investigations of ICs. It is well known that colloidal metal particles are forming if a metal is evaporated in a residual gas atmosphere. These are good IR absorbers ('silver black' or 'gold black'), which are used in industrial IR detectors [7]. However, these layers are electrically conducting. Therefore they are not appropriate for IC investigation with electrical stimulation, since they would provide a short at the terminals of the IC. We have found that colloidal bismuth is leading to high-ohmic black layers, whose conductivity is not significant anymore after a few days of storage in air [8]. These films are prepared by evaporating $600 \mathrm{mg}$ of $\mathrm{Bi}$ from a tantalum evaporation boat within $10 \mathrm{~s}$ at a residual air pressure of $200 \mathrm{~Pa}$ in a closed evaporation assembly, which provides a radiation shield between the evaporator and the specimen. More details to the preparation of these black thin films can be found in [8]. These films have a thickness of typically $10 \mu \mathrm{m}$ and provide an absorptance above $80 \%$ 
in the spectral range of 3 to $5 \mu \mathrm{m}$. They consist from fluffily-packed nanoscopic metal flakes, which can easily be removed from the surface in an ultrasonic water bath. Fig. 3 shows a $0^{\circ}$ image of the uncovered surface (a), the $0^{\circ}$ image of the black surface (b), and the power distribution (c) deconvoluted from (b) of a faulty region in a CMOS IC. Indeed, in the image of the blackened surface, the visible emissivity contrast is removed. Again, the deconvolution procedure leads to a considerable deblurring of the image. In this case, in the deconvoluted image certain residua of the emissivity contrast are visible, which are weak horizontal and vertical dark lines. They point to the fact that the absorptance of the black film was not $100 \%$. In this case, however, this residual emissivity contrast is not dominating, as it was in Fig. 1 (c). Hence it does not disturb here and may be even helpful to orientate on the surface of the IC.

\section{Conclusions}

It has been shown that spatial deconvolution of microscopic LIT images is possible, even in the presence of strong emissivity contrast, if the $0^{\circ} /-90^{\circ}$ image is used for deconvolution. This kind of representation of LIT results is advantageous, compared to the usual phase image, since it provides a better spatial resolution. Thus, the $0^{\circ} /-90^{\circ}$ image can be used in all cases where until now the phase image has been used, if thermal blurring is degrading the effective spatial resolution. This kind of inherent compensation of the emissivity contrast is easier and more effective than blackening the surface or the usual separate measurement of the emissivity image, since it does not require any extra measurement and provides a perfect emissivity compensation. However, its application is limited to microscopic problems, where the thermal $-90^{\circ}$ signal is nearly homogeneous across the investigated area.

\section{Acknowledgement}

This work was supported by EU project ENK6-CT-2001-00573 (PORTRAIT). Many thanks to EM Microelectronic-Marin SA (Marin, Switzerland) for providing the sample for Fig. 3.

\section{REFERENCES}

[1] MALDAGUE (X.P.V.). - Theory and practice of infrared technology for nondestructive testing. New York, Wiley, 2001.

[2] BREITENSTEIN (O.) and LANGENKAMP (M.). - Lock-in thermography - basics and use for functional diagnostics of electronic components. Berlin, Springer, 2003.

[3] BREITENSTEIN (O.). - Lock-in IR thermography for functional testing of solar cells and electronic devices. QIRT Journal 1 (2004) 151.

[4] CREPEL (O.), BEAUDOIN (F.), DANTAS DE MORAIS (L.), HALLER (G.), GOUPIL (C.), PERDU (P.), DESPLATS (R.), and LEWIS (D.). - Backside hot spot detection using liquid crystal microscopy. Microelectronics Reliability 42 (2002) 1741

[5] KOLODNER (P.) and TYSON (J.A.). - Microscopic fluorescent imaging of surface temperature profiles with $0.01^{\circ} \mathrm{C}$ resolution. Appl. Phys. Lett. 40 (1982) 782.

[6] CARSLAW (H.S.) and JAEGER (J.C.). - Conduction of heat in solids. Oxford, Clarendon, 1959.

[7] NORKUS (V.), GERLACH (G.), and HOFFMANN (G.). - Process technologies for high-resolution infrared detectors based on LiTaO3. Proc. SPIE 3892 (1999) 233.

[8] GRADHAND (M.) and BREITENSTEIN (O.). - Preparation of nonconducting infrared-absorbing thin films. Rev. Sci. Instr. 76 (2005) 053702. 